\title{
Multi-cell Massive MIMO Network Optimization towards power consumption in Suburban Scenarios
}

\author{
Michel Matalatala ${ }^{1}$, Margot Deruyck ${ }^{1}$, Emmeric Tanghe ${ }^{1}$, Sotirios Goudos ${ }^{2}$, Luc Martens ${ }^{1}$ and \\ Wout Joseph ${ }^{1}$ \\ ${ }^{1}$ Department of Information Technology, Ghent University/IMEC, Ghent, Belgium \\ michel.matalatala@ugent.be \\ ${ }^{2}$ Department of Physics Aristotle University of Thessaloniki, Thessaloniki, Greece \\ sgoudo@physics.auth.gr
}

\begin{abstract}
Future wireless communication networks are challenged to meet the demand for reliable high throughput while improving both the spectral and energy efficiency, by using appropriate technology like massive MIMO. In this paper, we propose a simulation-based method to design low power multi-cell multi-user massive MIMO network by optimizing the positions of the base stations. Two realistic outdoor suburban areas have been considered in Ghent, Belgium (Europe) and Kinshasa, the Democratic Republic of Congo (Africa), in which the power consumption, the energy efficiency, the network capacity and the multiplexing gain are investigated and compared with LTE networks. The results of the simulations demonstrated that massive MIMO networks provide better performance in the crowded scenario where user's mobility is relatively low. A massive MIMO BS consumes 5-8 times less power than the LTE networks, with a pilot reuse pattern of 3 that helps obtaining a good tradeoff between the higher bit rate requested and the low power requirements in cellular environment.

Index Terms-5G, massive MIMO (Multiple Input Multiple Output), capacity-based deployment tool, coverage, power consumption, energy efficiency.
\end{abstract}

\section{INTRODUCTION}

The introduction of multiple input multiple output (MIMO) and carrier aggregation technologies in the $4^{\text {th }}$ generation of the mobile networks (4G) lead to more energy-efficient networks, in comparison with the $3^{\text {th }}$ generation of mobile networks (3G) [1]. Recently, much attention have been garnered to the massive MIMO as an indispensable technology for the future $5^{\text {th }}$ generation of mobile wireless networks (5G) [2]. It can support higher spectral efficiencies to cope with the requested high throughput, and provide impressive energy efficiency performance at the same time [3], [4]. In fact, massive MIMO which uses very large number of antennas at the base stations (BS) to serve many users simultaneously at the same time-frequency resource, allow the transmission of user data at very low power per antenna element, reducing the energy consumption of the BS at the same time. It provides the same quality of service with less radiated power [5]-[7] compared to $4 \mathrm{G}$.

In developing countries like the Democratic Republic of Congo, energy harvesting is a big challenge because of the grid power capabilities that cannot supply enough energy to meet the increasing demand of the industries, mainly the wireless mobile communication ones. These latter rely on generators to compensate the grid energy shortage, with negative impact on the environment. So, implementing massive MIMO in such environment would be beneficial for the operators since they can build up cost-effective wireless networks and drastically reduce both their network capital and operational expenditures (CAPEX and OPEX) thanks to the important energy saving.

In this study, we propose numerical simulations for realistic designs of massive MIMO-based 5G cellular networks that provide higher throughput to the users, with low power consumption thanks to on optimal deployment of base stations within suburban areas in Kinshasa, the Democratic Republic of Congo and Ghent, Belgium. We use the capacity-based network deployment tool developed in [8] to build energyefficient $5 \mathrm{G}$ cellular networks with an optimal number of the base stations needed to guarantee the requested quality of service (QoS). The results of the simulations are analyzed and compared with the LTE networks.

Some related works in [9], [10] addressed the design of macro-cellular massive MIMO networks where the energy efficiency (EE) is maximized for a given coverage of the users $(95 \%)$. Based on an assumed number of network cells, the maximal EE is achieved with a hundred of BS antennas serving tens of users in parallel, which match well with the massive MIMO concept. The main contribution of this work consists in optimizing the sets of realistic possible locations of BSs retrieved from mobile operators in Kinshasa and Ghent, respectively so that the designed cellular massive MIMO networks meet the requested high bit rate, the coverage probability (95\% of the users) and the power consumption requirements. To the best of our knowledge, none of the related existing works investigated the optimization of BS positions, in the context of multi-cell massive MIMO environment.

The remainder of this paper is organized as follows. Section II describes the main assumptions and the massive MIMO models that are used in the simulations. In Section III, the method to deploy the optimal number of base stations together with the proposed algorithm is provided and finally, Section IV presents the numerical results obtained with the simulations with respect to the assumed massive MIMO scenarios. We then conclude the study in Section V. 


\section{Multi-Cell Massive MiMO system MOdel}

In this study, the massive MIMO system model consists of two suburban scenarios in Africa and Europe: a suburban area of $5.2 \mathrm{~km}^{2}$ in Kinshasa $(\mathrm{KN})$, Democratic Republic of Congo (Fig. 1) and a suburban area of $6.85 \mathrm{~km}^{2}$ in Ghent (GH), Belgium (Fig. 2). In the two suburban areas, 75 and 59 base stations are deployed in $\mathrm{GH}$ and $\mathrm{KN}$ respectively, each equipped with a large number of antennas, $M$, operating phase-coherently. Each base station serves many single-antenna users $K$ simultaneously in its associated cell. However, the different base stations do not cooperate. Each user is associated and covered by one of the BSs. The

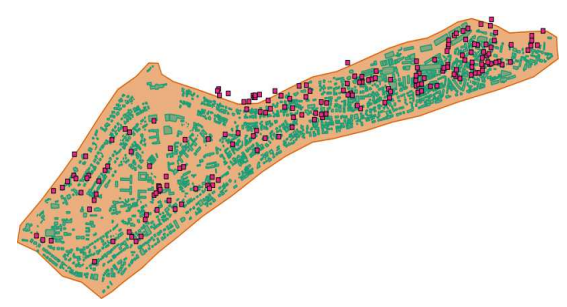

Figure 1: Selected area in Gombe, Kinshasa and the possible locations of the base stations

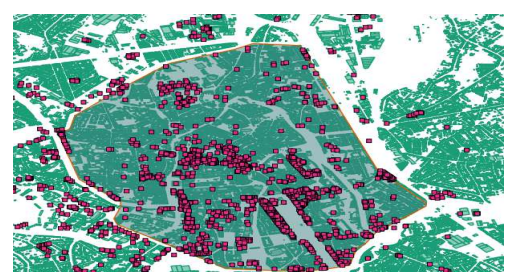

Figure 2: Selected area in Ghent, Belgium and the possible locations of the base stations

maximum number of served users $K_{\max }$ at peak hours is estimated to 147 users and 224 users for Kinshasa and Ghent, respectively, based on the confidential data provided by the mobile operators.

In both environments, we consider that the network operates in time division duplex (TDD) mode to benefit from the channel reciprocity properties. Here, we denote the coherence interval length as $\tau_{c}$, expressed in symbols and modeled as the product of the coherence time $\tau_{t}$ in milliseconds and the coherent bandwidth $\tau_{B}$ in kilohertz. The number of symbols $\tau_{p}$ reserved for the channel estimation is chosen such that $\tau_{p} \leq \tau_{c}$; the remaining $\tau_{c}-\tau_{p}$ symbols are dedicated for the data transmission, mainly in the downlink (DL).

The effects of inter-cell interference caused by the pilot contamination phenomenon inherent to the TDD multi-cell environment are captured by means of a different pilot reuse pattern: 1,3 and 7. The other sources of interference are not considered in this study. Therefore, under the favorable propagation conditions requiring the mutual orthogonality of the propagation channels between the BS antenna arrays and the users, the signal to interference and noise ratio (SINR) experienced by the $k^{t h}$ user in Cell $l$ is modeled similarly to [11]:

$$
S I N R_{l k}=\frac{M \alpha_{M R T} p_{p} p_{d l k} \beta_{l k l}^{2}}{M \alpha_{M R T} p_{p} \sum_{j \neq l}^{L} p_{d j k} \beta_{j k l}^{2}+\sigma_{n}^{2}}
$$

With $\alpha_{M R T}$ the normalization constant considered to satisfy the transmit power constraint at the BS [12]; $p_{p}$ is the power of the pilots (in $\mathrm{W}$ ) and $p_{d l k}$ is the DL power of the serving base station (in W) in cell $l$ towards user $k ; p_{d j k}$ is the DL power of the interfering BS (in W) in cell $j$ towards user $k$ in cell $l$; $\beta_{j k l}$ represents the large-scale fading between the base sation in $j^{\text {th }}$ cell and the user $k$ in $l^{\text {th }}$ cell that takes into account the geometric attenuation, shadow fading and path loss and $\sigma_{n}^{2}$ is the noise variance. In the same way, the capacity of the cell is modeled as follows [11]:

$$
C_{l}=B \cdot \sum_{k=1}^{K} R_{l k}(\text { bits } / s)
$$

Where $\mathrm{B}$ is the bandwidth of the channel (in $\mathrm{Hz}$ ), $K$ the number of users simultaneously served by the cell $l$ and $R_{l k}$ the achievable rate of the $k^{t h}$ user in cell $l$ of a multicell system under imperfect channel state information (CSI) defined by [11], [13]:

$$
R_{l k}=\gamma^{D L}\left(1-\frac{\tau_{p}}{\tau_{c}}\right) \log _{2}\left(1+S I N R_{l k}\right)(\text { symbols } / s / H z)
$$

With $S I N R_{l k}$ defined in (1) and $\gamma^{D L}$ is the portion of the coherence interval dedicated to DL transmission $\left(\gamma^{D L}=\right.$ $50 \%$ [7]).

\section{MethoD}

We consider the Massive MIMO setup in which the operating frequency is set at $3.70 \mathrm{GHz}$, the bandwidth at 20 $\mathrm{MHz}$ (below $6 \mathrm{GHz}$ ). For realistic and practical reasons, we assume the BS uses multiple antennas $(16,32,48,64$ and 256 ) and the user equipment has one single transmit antenna. Besides the $4 \mathrm{G}$ reference network investigated in [1], relevant massive MIMO scenarios are considered [14], [15]: the open exhibition scenario (crowded environment with almost static users) with users requesting $20 \mathrm{Mbps}$ on average for their applications (live streaming) and the dense urban information society scenario (fast users) with applications (real time video gaming) requiring $300 \mathrm{Mbps}$ on average per user. In both scenarios, we use a coherence bandwidth of $210 \mathrm{kHz}$ and the coherence time is set to 1 and $2 \mathrm{~ms}$ for the high user mobility and the low user mobility scenarios, respectively [7].

For each scenario assumed, we have to execute multiple simulations (40 simulations in total) to ensure a good estimation of the parameters investigated: the number of BS, the power consumption (PC), the energy efficiency (EE), the BS capacity and the multiplexing gain (MG) which denotes the number of users that can be served simultaneously by a BS during the TDD time slot. A uniform distribution is assumed 
for the location of each user within the areas of interest so that each location has the same chance to be chosen as a user location [1]. Similarly, we assume that the bit rates are uniformly distributed since the coherence beamforming gain (that grows with $M$ ) can help provide enough Signal to Noise Ratio (SNR) to enable higher bit rates for the users. Based on these distribution functions, we generate 40 traffic files that contain the maximum number of simultaneous active users, the location of the users in the area of interest and the required bit rates (based on the application).

We propose a simulation-based tool, developed in java, that we denote Capacity-based network deployment tool, which optimizes the initial set of base stations deployed by the operators in the area of interest based on the BS-user association algorithm with respect to the following optimization constraints: minimize the power consumption of the obtained network, respond to the instantaneous bit rates requested by the users and provide a coverage to at least $95 \%$ of the users. In [8], the same tool has been used but with beamforming capability only. Here, in addition to beamforming, we implement the massive MIMO requirements: the new bit rate distribution to comply with the user's $5 \mathrm{G}$ applications, the sum spectral efficiency described in (2), the 3GPP LTE Rel. 8 path loss model and the power consumption model. Both the 3GPP LTE Rel. 8 path loss model and the power consumption one have been proposed for massive MIMO in [15]. They appear to be more realistic and lead to fair comparison with the $4 \mathrm{G}$ LTE.

Besides, additional files containing the set of possible locations of the BSs, the geospatial informations of the environments, the link budget parameters [15] and the PC values [15], [16] of the BSs' sub-components are separately added as inputs to the tool that simulates different scenarios of the massive MIMO system. The algorithm implemented in the tool calculates the path loss between the user and all the BSs in the initial set of base stations. The BS with the lowest path loss and enough capacity to support the bit rates demanded by the user is enabled and associated with the user, on the condition that this lowest path loss is lower than the maximum allowable path loss (MAPL). For a new user, the algorithm checks the possibility to connect him with an existing active BS. If it is not possible, the BS power is gradually incremented until the association conditions are met. If the maximum transmit power of the BS is reached and it is still not possible to connect the user to the existing active BS, a new BS will be enabled. In this latter case, the algorithm assesses the possibility to transfer the already connected users to this newly activated BS as they may experience lower path loss and the old BS is deactivated automatically if it is left empty. If no BS can be enabled or all BSs are active, the user cannot be covered. The operation is repeated for all the users. The objective is to activate the least possible BSs and the obtained subsets of active BSs with their respective placements are then optimal. The results of the simulations are then compared with the 4G LTE reference network under similar deployments: same environments, same BS optimization algorithm and energy efficiency model [8].

The values of the main link budget parameters used for the computation of the algorithm are obtained from [8], [15]. The power consumption model approach proposed takes into account the way the PC of the main components or sub-components are influenced by some parameters like: the bandwidth, the frequency, the quantization resolution, the number of antennas, the load factor, the streams and the spectral efficiency. The values are obtained based on the PC values at a reference operating point $(20 \mathrm{MHz}$, single antenna, 24 bit-quantization) and the scaling coefficients that account for the current operating parameters [15], [16]. The EE metric defined in [8] taking into account multiple network performance parameters such as the bandwidth, the bit rate, the coverage and the capacity is used here, in $\left[\mathrm{km}^{2} \cdot \mathrm{Mbps} / W\right]$ :

$$
E E=\frac{A \cdot B \cdot U}{P_{e l}}
$$

where $A$ is the area covered by the BS (in $k m^{2}$ ), $U$ is the number of served users, $B$ is the bit rate based on the base station (in $M b p s$ ) and $P_{e l}$ is the PC of the base station (in Watt). The higher the EE value, the more energy-efficient the network is.

\section{RESULTS AND DISCUSSIONS}

Here, we discuss the results of the simulations of the different 5G massive MIMO networks with respect to the scenarios described in Section III. Table I shows the results of the simulations in terms of the $95^{\text {th }}$ percentile of each investigated parameter, calculated over the 40 simulations. The standard deviation $\sigma_{P C}$ and $\sigma_{E E}$ and the confidence intervals $C I_{P C}$ and $C I_{E E}$ of the power consumption and the energy efficiency, respectively, are estimated. $\sigma_{P C}$ varies from $0.03 \mathrm{~kW}$ to $0.16 \mathrm{~kW}$ and from $0.034 \mathrm{~kW}$ to 0.19 $\mathrm{kW}$, while $\sigma_{E E}$ varies from $128.32\left[\mathrm{~km}^{2} \cdot M b p s / W\right]$ to $1601.6\left[\mathrm{~km}^{2} \cdot M b p s / W\right]$ and from $175.7\left[\mathrm{~km}^{2} \cdot M b p s / W\right]$ to $1652.4\left[\mathrm{~km}^{2} \cdot M b p s / W\right]$ for Kinshasa $(\mathrm{KN})$ and Ghent $(\mathrm{GH})$ environment, respectively. The uncertainties of the calculated percentiles are small enough to consider that our estimation are good. For instance, in all the scenarios considered, the uncertainty of the power consumption is between $0.008 \mathrm{~kW}$ and $0.048 \mathrm{~kW}$ for Kinshasa environment and between 0.01 $\mathrm{kW}$ and $0.06 \mathrm{~kW}$ for the Ghent scenario.

\section{A. Power Consumption}

From all the scenarios examined, the results show that massive MIMO BS consumes 5-8 times ( $\mathrm{KN}$ and GH, respectively) less power than the $4 \mathrm{G}$ reference scenario and provides almost 100-200 times more capacity. In fact, from the scenario with 256 antennas, a massive MIMO BS consumes only $172 \mathrm{~W}$, while the $4 \mathrm{G}$ base station needs $848 \mathrm{~W}$ to provide services to the users. The low-power consumption components used in massive MIMO base stations, with simpler architecture are the main contributors to this good performance. Moreover, the use of many antennas at the massive MIMO BS requires low output power level at each antenna. Therefore, the traditional power amplifier (PA) used in $4 \mathrm{G}$ is not needed here; instead, a predriver at the buffer stage of the front-ends will be preferred 
Table I: Simulation Results (95 percentile)

\begin{tabular}{|c|c|c|c|c|c|c|c|}
\hline Scenarios & $\begin{array}{c}\text { Pilot reuse } \\
\text { Pattern }\end{array}$ & $\begin{array}{l}\text { MIMO } \\
\text { Config }\end{array}$ & $\begin{array}{c}\text { BS } \\
{[-]} \\
\text { KN / GH }\end{array}$ & $\begin{array}{c}\mathbf{P C} \\
{[\mathbf{k W}]} \\
\mathbf{K N} / \mathbf{G H}\end{array}$ & $\begin{array}{c}\text { BS Capacity } \\
\text { [Mbps] } \\
\text { KN / GH }\end{array}$ & $\begin{array}{c}\mathbf{E E} \\
{\left[k m^{2} \cdot M b p s / W\right]} \\
\mathbf{K N} / \mathbf{G H}\end{array}$ & $\begin{array}{c}\text { MG } \\
{[-]} \\
\text { KN / GH }\end{array}$ \\
\hline 4G LTE & & - & $23 / 33$ & $19.52 / 46.5$ & $129.34 / 449.48$ & $4.75 / 14.57$ & - \\
\hline \multirow{15}{*}{ Open Exhibition } & \multirow{5}{*}{1} & $16 \times 1$ & $25 / 35$ & $0.96 / 1.34$ & $2622.89 / 25451.17$ & $1810.24 / 3794.61$ & $5.78 / 6.2$ \\
\hline & & $32 \times 1$ & $19 / 29$ & $0.9 / 1.37$ & $2642.89 / 21199.36$ & $2068.22 / 4197.21$ & $7.65 / 7.66$ \\
\hline & & $64 \times 1$ & $15 / 22$ & $0.98 / 1.43$ & $2660.9 / 16047.67$ & $1979.91 / 4196.41$ & $9.80 / 10.18$ \\
\hline & & $128 \times 1$ & $12 / 17$ & $1.21 / 1.71$ & $2660.9 / 12411.79$ & $1652.05 / 3575.03$ & $12.25 / 13.18$ \\
\hline & & $256 \times 1$ & $10 / 13$ & $1.73 / 2.24$ & $2340.7 / 9497.5$ & $1023.11 / 2789.83$ & $14.63 / 17.23$ \\
\hline & \multirow{5}{*}{3} & $16 \times 1$ & $24 / 35$ & $0.92 / 1.34$ & $2302.63 / 96227.31$ & $1643.98 / 3755.19$ & $6.01 / 6.13$ \\
\hline & & $32 \times 1$ & $20 / 29$ & $0.94 / 1.37$ & $50653.45 / 81049.58$ & $1742.25 / 4186.2$ & $7.35 / 7.69$ \\
\hline & & $64 \times 1$ & $15 / 22$ & $0.98 / 1.43$ & $38408.1 / 62368.93$ & $1753.22 / 4208.08$ & $9.8 / 10.18$ \\
\hline & & $128 \times 1$ & $12 / 16$ & $1.21 / 1.61$ & $30970.92 / 45835.2$ & $1451.34 / 3777.02$ & $12.25 / 14$ \\
\hline & & $256 \times 1$ & $10 / 12$ & $1.72 / 2.08$ & $25956.66 / 34926.36$ & $1022.66 / 2980.73$ & $14.7 / 18.51$ \\
\hline & \multirow{5}{*}{7} & $16 \times 1$ & $25 / 35$ & $0.96 / 1.34$ & $41589.99 / 57994.01$ & $1825.92 / 3764.66$ & $5.8 / 6.17$ \\
\hline & & $32 \times 1$ & $20 / 28$ & $0.95 / 1.33$ & $35496.52 / 49624.49$ & $1977.01 / 4342.37$ & $7.26 / 7.94$ \\
\hline & & $64 \times 1$ & $15 / 22$ & $0.98 / 1.43$ & $28219.20 / 41114.07$ & $1964.34 / 4257.15$ & $9.73 / 10.18$ \\
\hline & & $128 \times 1$ & $12 / 17$ & $1.21 / 1.72$ & $23566.45 / 33582.19$ & $1648.81 / 3567.66$ & $12.25 / 13.1$ \\
\hline & & $256 \times 1$ & $9 / 13$ & $1.55 / 2.24$ & $18447.05 / 26645.74$ & $1302.38 / 2771.68$ & $16.33 / 17.23$ \\
\hline \multirow{10}{*}{ Information Society } & \multirow{5}{*}{3} & $16 \times 1$ & $20 / 39$ & $0.77 / 1.49$ & $50149.62 / 79781.79$ & $39692.73 / 63288.86$ & $7.31 / 5.74$ \\
\hline & & $32 \times 1$ & $20 / 38$ & $0.94 / 1.79$ & $50653.45 / 78481.91$ & $32316.31 / 52624.4$ & $7.35 / 5.89$ \\
\hline & & $64 \times 1$ & $19 / 37$ & $1.23 / 2.41$ & $48650.26 / 76943.04$ & 24668.97 / 39194.2 & $7.73 / 6.05$ \\
\hline & & $128 \times 1$ & $19 / 36$ & $1.91 / 3.63$ & 49037.29 / 75232.26 & $15928.43 / 26010.16$ & $7.73 / 6.22$ \\
\hline & & $256 \times 1$ & $18 / 36$ & $3.09 / 6.2$ & 46721.98 / 75496.39 & $9844.90 / 15230.01$ & $8.17 / 6.22$ \\
\hline & \multirow{5}{*}{7} & $16 \times 1$ & $30 / 45$ & $1.15 / 1.72$ & $49709.15 / 74563.73$ & $26583.03 / 54855.52$ & $4.9 / 4.98$ \\
\hline & & $32 \times 1$ & $29 / 44$ & $1.37 / 2.08$ & $51213.88 / 77703.82$ & 22287.11 / 45448.34 & $5.06 / 5.09$ \\
\hline & & $64 \times 1$ & $25 / 37$ & $1.63 / 2.41$ & 46720.53 / 69146.39 & $18748.41 / 39194.2$ & $5.88 / 6.05$ \\
\hline & & $128 \times 1$ & $24 / 36$ & $2.42 / 3.64$ & $47132.90 / 70895.74$ & $12610.01 / 25938.11$ & $6.12 / 6.2$ \\
\hline & & $256 \times 1$ & $24 / 36$ & $4.13 / 6.2$ & $49192.13 / 73788.19$ & $7383.67 / 15230.01$ & $6.12 / 6.22$ \\
\hline
\end{tabular}
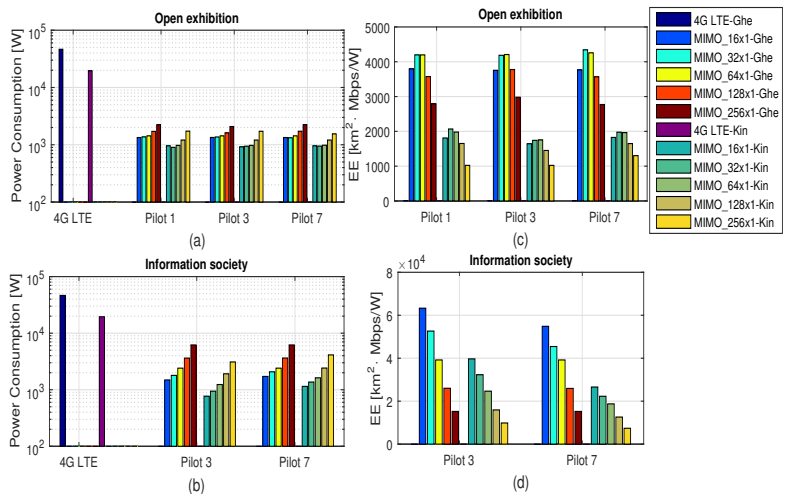

Figure 3: Power consumption and EE comparison for both Open Exhibition and Information society scenarios in Kinshasa and Ghent (Pilot 1, 3 and 7 refers to pilot reuse pattern 1,3 and 7 respectively.)

in order to comply with this low output power level requirement for 5G. Fig. 3 (a) and (b) show almost the same behavior, in both environments, in terms of power consumption which scales with the number of BS antennas. In scenario II (Fig. 3 (a)), when the number of antennas increases from 16 to 256 , the power consumption increases by $68 \%$ and $67 \%$ in $\mathrm{GH}$ and $\mathrm{KN}$ respectively, while in scenario III (Fig. 3 (b)), the increase is more pronounced. In facts, when the BS is equipped with 256 antennas, the power consumption is 4 times higher compared to the case where 16 antennas are used, in both Ghent and Kinshasa environments. This is mostly attributed to the number of RF chains behind the antennas. There are as many antennas as the number of associated RF chains. But this power consumption increase still remains lower than that of the $4 \mathrm{G}$ reference network. The massive MIMO network $(256 \times 1)$ in Ghent consumes almost 3 times more power than the Kinshasa networks (256x1) (Table I). This can be explained by the geometry of the Ghent environment which requires more BSs to compensate the non line of sight propagations imposed by the high number of buildings so as to provide coverage to at least $95 \%$ of the users. There are 30\% more buildings in Ghent environment than in Kinshasa.

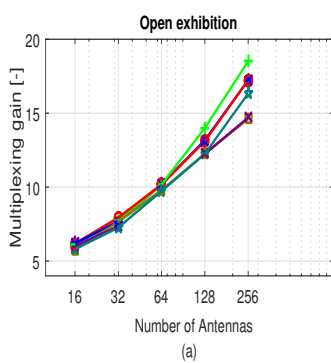

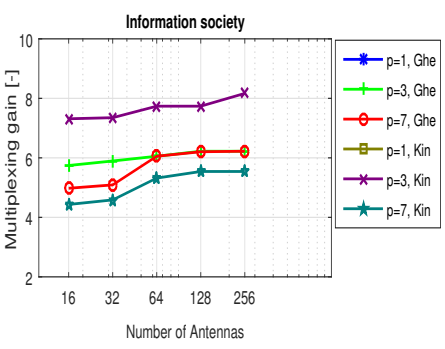

Figure 4: Effects of pilot contamination (KN and $\mathrm{GH})$

\section{B. Energy Efficiency}

Fig. 3 (c) and (d) shows a degradation of the energy efficiency as the circuit power increases with the number of massive MIMO BS antennas. The more antennas, the less energy efficient the massive MIMO network is since the power consumption grows with the number of BS antennas. From the same figure, an interesting observation can be made: the massive MIMO network in Ghent is 2 times more energyefficient than the one deployed in Kinshasa. The number of active users at the peak hours is the main contributing factor (224 active users for Ghent and 147 users for Kinshasa). In fact a higher number of active users to be served at peak hours increase the probability to simultaneously serve more users (at the same time-frequency resource) within the serving cell whose capacity is increased at the same time. Massive MIMO networks outperform the $4 \mathrm{G}$ reference network in terms of energy efficiency. They are 400 times more energy efficient than $4 \mathrm{G}$ networks and this performance is achieved thanks to the number of simultaneous users that can be multiplexed and the low-power consumption equipments they are equipped with. 


\section{Multiplexing gain and pilot contamination}

Fig. 4 shows that the multiplexing gain is important when the number of antennas at the base stations increases, despite the effect of the inter-cell interference (pilot contamination). Even when the reuse pattern is set to 1 (most interfering situation), the benefit of the spatial multiplexing gain is still noticeable through the increase of the served users. In scenario II (open exhibition), the multiplexing gain is multiplied by 3 when the number of antennas grows from 16 to 256 in both Ghent and Kinshasa environments, while in scenario III (Dense urban information society), it is increased by $25 \%$ when the number of antennas vary from 16 to 256 (Fig. 4).

In both Ghent and Kinshasa, when comparing the two scenarios investigated, there are almost 3 times more users served by multiplexing in scenario II than in scenario III (table I). This is mainly explained by the mobility of the users: in an environment with moderate mobility of the users like an open exhibition, it is required to use a longer coherence interval ( $2 \mathrm{~ms}$ in our study) that provides more room for longer pilots (210 pilots in our study) and give possibilities to serve many users, while the high mobility environment requires a shorter coherence interval (1 $\mathrm{ms}$ in our study). Fig. 4 shows that the Ghent environment presents better performance in terms of multiplexing gain, compared to that of the Kinshasa environment. The massive MIMO network in Ghent can simultaneously serve $20 \%$ more users than the Kinshasa one for the open exhibition scenario and 5\% more users for the information society one.

In terms of base station capacity, the better results are achieved with the pilot reuse pattern of 3 compared to the pattern reuse of 7 (Table I): it provides 23\% more capacity for scenario II and 3\% more capacity for scenario III. The number of pilots is divided by 7 in the reuse pattern of 7 since they have to be shared between all the non-interfering cells belonging to this pattern. With the reuse pattern 3 , the number of pilots is divided only by 3 . This means that massive MIMO networks with a pilot reuse pattern of 3 can serve more users than those with a reuse pattern of 7 .

\section{CONCLUSION}

In this study, we propose a simulation-based method to design a massive MIMO network with optimized BS positions that meets the higher bit rate and low power requirements. We investigate two suburban areas in Ghent, Belgium (Europe) and Kinshasa, the Democratic Republic of Congo (Africa) in which we consider two scenarios for the deployment of massive MIMO networks at $3.75 \mathrm{GHz}$ : a crowded scenario like open exhibition or stadium with slow users and a urban information society, less crowded but with high mobility. We show that the use of very large number of antennas at the BS makes it possible for the massive MIMO network to achieve the same performance as a $4 \mathrm{G}$ reference network in terms of coverage while consuming 5-8 times less power and providing 100-200 times more capacity in Ghent and Kinshasa, respectively. In both environments of study, the results reveal that massive MIMO is more appropriate for crowded scenarios since it provides uniformly good services to 2-3 times more users simultaneously, compared to the information society scenario. Moreover, Ghent environment simultaneously serves 20\% (crowded scenario) and 5\% (information society) more users while consuming 3 times more power, in comparison to Kinshasa. The pilot reuse pattern of 3 appears to be the optimum one to ensure a trade-off between higher capacity and low power consumption requirements.

\section{ACKNOWLEDGMENT}

We conducted this work with the financial support of the Research Foundation at UGent (BOF-UGent) under the grant agreement number 01W0014. Margot Deruyck and Emmeric Tanghe are post-doctoral fellows of the FWO-V (Research foundation-Flanders)

\section{REFERENCES}

[1] M. Deruyck, W. Joseph, E. Tanghe, and L. Martens, "Reducing the power consumption in LTE-advanced wireless access networks by a capacity based deployment tool," Radio Science, vol. 49, pp. 777-787, September 2014.

[2] M. Hossain, C. Cavdar, E. Björnson, and R. Jäantti, "Energy-efficient load-adaptive massive MIMO," in 2015 IEEE Globecom Workshops (GC), San Diego, CA, USA, December 2015, pp. 1-6.

[3] T. Marzetta, "Noncooperative cellular wireless with unlimited numbers of base station antennas," IEEE Transactions Wireless Communications, vol. 9, no. 11, pp. 3590-3600, November 2010

[4] F. Boccardi, R. Health, A. Lozano, T. Marzetta, and P. Popovski, "Five disruptive technology directions for 5G," IEEE Communications Magazine, vol. 52, no. 2, pp. 74-80, February 2014.

[5] E. Larsson, O. Edfors, F. Tufvesson, and T. Marzetta, "Massive MIMO for next generation wireless systems," IEEE Communication Magazine, vol. 52, no. 2, pp. 186-195, February 2014.

[6] E. Bjornson, E. Larsson, and T. Marzetta, "Massive MIMO: ten myths and one critical question," IEEE Communications Magazine, vol. 54, pp. 114-123, February 2016.

[7] T. Marzetta, E. Larsson, H. Yang, and H. Quoc, "Fundamentals of Massive MIMO," Cambridge University Press, 2016.

[8] M. Matalatala, M. Deruyck, E. Tanghe, L. Martens, and W. Joseph, "Performance evaluation of $5 \mathrm{G}$ millimeter-wave cellular access networks using a capacity-based network deployment tool," Mobile Information Systems, vol. 2017, p. 11, January 2017.

[9] H. Ngo, E. Larsson, and T. Marzetta, "Energy and spectral efficiency of very large nultiuser MIMO systems," IEEE Transactions on Wireless Communications, vol. 61, pp. 1436-1449, April 2013.

[10] H. Young and T. Marzetta, "Total energy efficiency of cellular large scale antenna system multiple access mobile networks," in IEEE Online Conference on Green Communications(OnlineGreenComm), Piscataway, NJ, USA, October 2013, pp. 27-32.

[11] S. Jabbar and Y. Li, "Analysis and evaluation of performance gains and tradeoffs for Massive MIMO systems," Applied Sciences, vol. 6, September 2016.

[12] H. Ngo, E. Larsson, and T. Marzetta, "Massive mu-mimo downlink TDD systems with linear precoding and downlink pilots," in 2013 51st Annual Allerton Conference on Communication, Control and Computing, Monticello, IL, USA, 2-4 October 2013, pp. 293-298.

[13] T. Chien, E. Bjornson, and E. Larsson, "Joint power allocation and user association optimization for Massive MIMO systems," IEEE Transactions on Wireless Communications, vol. 15, pp. 6384-6399, September 2016.

[14] METIS project, "in scenarios, requirements and KPIs for 5G mobile and wireless system, deliverable D1.1," May 2013.

[15] MAMMOET, "Massive MIMO for efficient transmission: Deliverables 1.1, systems scenarios and requirements specifications," 2014.

[16] C. Desset, B. Debaillie, and F. Louagie, "Modeling the hardware power consumption of large scale antenna systems," in 2014 IEEE Online Conference on Green Communications, Monticello, IL, USA, 2-4 October 2013, pp. 293-298. 